\title{
LÍNEAS DE FUERZA DE LO FANTÁSTICO EN LAS CREADORAS ESPAÑOLAS EN EL SIGLO XXI ${ }^{1}$
}

\author{
LINES OF FORCE OF THE FANTASTIC \\ IN SPANISH WOMEN WRITERS IN THE $21^{\text {st }}$ CENTURY \\ Natalia ÁLVAREZ MÉNDEZ \\ Universidad de León \\ natalia.alvarez@unileon.es
}

\begin{abstract}
Resumen: Este estudio preliminar funciona como contextualización sintética de la sección monográfica, planteando las premisas iniciales y una fundamentación teóricocrítica. La pretensión es doble: plasmar la necesidad de defensa de la existencia de una tradición femenina en el ámbito de la literatura de lo irreal y sintetizar los aspectos esenciales que conviene considerar en el análisis de dicha creación en el siglo XXI, entre ellos el hibridismo genérico, la significación del espacio doméstico y corporal, la relevancia de las voces narradoras de mujer, el protagonismo femenino con una marcada agencia, el modo de abordar la monstruosidad, así como las perspectivas feministas y de compromiso social y político.
\end{abstract}

Palabras clave: Narrativa española femenina. Narrativa fantástica. Narrativa de lo insólito. Monstruosidad femenina. Feminismo.

\begin{abstract}
This preliminary study serves as a brief contextual framework of the following monographic section, including its cornerstones and a theoretical background. It has two goals: the claim of a female tradition in the field of unreal literature, and a synthesis of the essential aspects which must be taken into account in the analysis of this type of writing in the $21^{\text {st }}$ Century, including genre hybridity, domestic and corporeal space signification, the relevance of women's narrative, the prominence of female characters as agents, the ways in which monstrosity is tackled, as well as feminist, social and politically engagée perspectives.
\end{abstract}

Keywords: Spanish women's narrative. Fantastic fiction. Fantasy fiction. Female monstrosity. Feminism.

\footnotetext{
${ }^{1}$ Este número monográfico se inscribe en los proyectos de investigación Lo fantástico en la cultura española contemporánea (1955-2017): narrativa, teatro, cine, televisión, cómic y radio, del Ministerio de Economía, Industria y Competitividad (FFI2017-84402-P, I.P. Dr. David Roas), y Estrategias y figuraciones de lo insólito. Manifestaciones del monstruo en la narrativa en lengua española (de 1980 a la actualidad, del Ministerio de Ciencia, Innovación y Universidades (PGC2018-093648-B-I00, I.P. Dra. Natalia Álvarez Méndez).
} 


\section{PREÁMBULO}

Las aproximaciones a la narrativa no mimética de autoría femenina realizadas por diversos miembros del Grupo de Estudios sobre lo Fantástico (GEF), de la Universidad Autónoma de Barcelona, y del Grupo de Estudios literarios y comparados de lo Insólito y perspectivas de Género (GEIG), de la Universidad de León, nos han permitido en los últimos años constatar la necesidad de ampliar los estudios existentes sobre dicho corpus. Variados eventos científicos y académicos dedicados a la materia, así como publicaciones específicas sobre la misma ${ }^{2}$, han puesto en valor la obra de destacadas narradoras. No obstante, y a pesar de que se sigue incrementando el empleo de lo fantástico en la creación actual $^{3}$, todavía queda mucha labor de reivindicación que realizar. Así lo confirma la escasa presencia de las mujeres en los cánones y también en las investigaciones sobre la literatura ajena al realismo. Por tal motivo, sin soslayar el todavía vigente debate teórico sobre lo fantástico femenino que se retrotrae a la década de los setenta del siglo XX - a partir de estudios como los de Anne Richter (1977) y de Ellen Moers (1976), que derivan en perspectivas más recientes como las de Anne Cranny-Francis (1990), María Abrakova (2004) y Gloria Alpini (2009), entre otras - , ofreceremos algunas calas en autoras de diferentes generaciones y en obras significativas de nuestro actual panorama literario, sin perder de vista las especificidades que se vislumbran en la poética actual de las narradoras españolas en su conjunto.

De modo previo a ese trabajo en equipo que tiene como resultado el presente monográfico, quiero realizar una aportación inicial sobre la categoría estética que nos ocupa y sus implicaciones, así como establecer algunas consideraciones sobre las líneas de fuerza de lo fantástico en la autoría femenina.

\section{LA LITERATURA DE LO IRREAL: DEFENSA DE LO FANTÁSTICO Y DE UNA TRADICIÓN FEMENINA}

Hace casi una década, abordé la coordinación de un monográfico para delimitar la impronta de lo fantástico en la cultura española del siglo XXI, centrando la atención en la vitalidad del género y en las características de su poética - motivos, estructuras y lenguaje - en narrativa, cine, televisión y cómic (Álvarez Méndez, 2013). La intención principal era poner de relieve la riqueza de esta categoría en cuanto a potencialidad significativa y a construcción narrativa:

Las inquietantes fracturas y perturbaciones de nuestra cotidianidad se filtran mediante la presentación de la convivencia de órdenes o dimensiones que colisionan con las certezas del individuo y que ponen de relieve la problemática de la identidad y de la crisis del yo

\footnotetext{
${ }^{2}$ Entre las más recientes, véanse Patricia García (2019), Teresa López-Pellisa (2019), Carmen Alemany Bay y Cecilia Eudave (2020) y David Roas (2020).

${ }^{3}$ No solo en la literatura, también en otras manifestaciones artísticas y en los medios de masas del siglo XXI, por lo que será conveniente ampliar en un futuro el espectro abordado en el presente monográfico.
} 
que aboca a su disolución. Asimismo, las nuevas vías de expresión de lo fantástico se ofrecen a través de mundos paralelos, bucles temporales, viajes en el tiempo, predeterminación, metalepsis, fantasmas, monstruos, metaficción, objetos imposibles, disolución de la identidad, el doble, presciencia, fusión de vigilia y sueños, metamorfosis $\mathrm{y}$ animalización, alteraciones de las capacidades cognitivas y un largo etcétera. Mediante transgresiones lingüísticas y una prosa cuidada - sea exacta, limpia, lírica o tamizada por el surrealismo, el humor negro, la ironía, la parodia, lo grotesco o el absurdo, según los casos-, se sorprende al lector con mínimas alteraciones que provocan la irrupción de lo fantástico en el contexto de un universo real (Álvarez Méndez, 2013: 196).

La extensión limitada de este tipo de estudios y el hecho de que no se buscara una visión panorámica completa sino calas en corpus destacados en el ámbito de las citadas manifestaciones artísticas, contribuyeron a que en el resultado final quedaran escasamente representadas las creadoras, una deuda que ahora se trata de reparar. Esto es así porque no solo sigue siendo necesario defender la escritura de la imaginación ante los persistentes prejuicios de un sector concreto de críticos, académicos, editoriales y lectores, sino que se muestra como indispensable reivindicar la autoría femenina.

Es cierto que cada vez parece más asumido el nítido anclaje en la realidad que presenta la literatura no mimética, que nos permite profundizar en una lectura crítica del mundo posmoderno y del individuo que lo habita. No en vano, ya en su origen como modalidad literaria específica, surge con esa capacidad:

La modalidad literaria que así se ha producido ha servido, en aquella contingencia histórica, para extender y ensanchar las áreas de la "realidad" humana interior y exterior que pueden estar representadas por el lenguaje y por la literatura y, aún más, para poner en discusión las relaciones que se establecen en cada época histórica, entre paradigma de realidad, lenguaje y nuevas estrategias de representación. Se trata, conviene añadir, de estrategias no sólo representativas, sino también cognoscitivas (Ceserani, 1999: 100).

El hecho de que lo fantástico y otras estéticas de lo insólito hayan sido etiquetadas como géneros populares o de consumo no impide que muchas de sus figuraciones narrativas se instalen en el ámbito de la literatura digna de observación. De tal modo, existe un interesante corpus de obras no realistas con gran calidad estética que traslucen el conocimiento de la tradición literaria, a la que incorporan nuevos recursos, motivos, estructuras y formas que han dado lugar a una poética de lo fantástico posmoderno de gran interés en pleno siglo XXI ${ }^{4}$. A esa vertiente estética se le suma, además, una vertiente ética, de compromiso, reflejada en inquietudes de naturaleza muy variada: epistemológicas, filosóficas, metafísicas, culturales, sociales, políticas, económicas y medioambientales. No nos enfrentamos, pues, a una literatura de evasión. Lo fantástico juega con la transgresión de la realidad, concebida esta como una construcción cultural desde paradigmas científicos y filosóficos, y cuestiona las leyes que la rigen, lo que aboca a poner en duda sus límites y a interrogarse acerca de su definición y su estabilidad. Pero

\footnotetext{
${ }^{4}$ Para más información sobre la poética de lo fantástico en la narrativa española posmoderna, véase Roas, Álvarez y García (2017).
} 
la única pretensión del texto fantástico no es la de interrogarse por la definición de lo real. Tal como asevera Rosalba Campra en su reciente estudio En los dobleces de la realidad. Exploraciones narrativas:

No creo que la lectura de un texto fantástico deba agotarse en este interrogante; por lo tanto, querría apuntar aquí a una actitud más o menos heterodoxa: la de plantear, a este tipo de textos, preguntas que no derivan de su naturaleza fantástica, sino que refieren al mundo de los significados posibles. Las páginas que siguen se proponen destacar las implicaciones ideológicas de algunos relatos que antologías, análisis críticos, historias de la literatura, clasifican como "fantásticos": sin duda lo son, [...]. Sin embargo, poniendo entre paréntesis esa clasificación, se podrían identificar distintas formas de reenvío a la historia, la política, la sociedad (2019: 80-81).

De ello se deriva la lógica de explorar la irradiación metafórica del elemento fantástico: "Si la intención autorial fuera denunciar una injusticia, una desigualdad, una violencia ¿para qué recurrir a modulaciones fantásticas cuando se dispone de la inmediatez del realismo? ¿Hay acaso algo, en lo fantástico, que permite 'decir más'?” (Campra, 2019: 93).

Sin embargo, conviene dar un paso más, pues la defensa de las modalidades no realistas no puede desligarse de una justa atención a la escritura de mujeres. Tiempo atrás, Rosemary Jackson (1981), empleando enfoques psicoanalíticos y sociológicos, reseñó la transgresión con la que lo fantástico cuestiona el orden simbólico normativo e institucionalizado:

The modern fantastic, the form of literary fantasy within the secularized culture produced by capitalism, is a subversive literature. It exists alongside the "real", on either side of the dominant cultural axis, as a muted presence, a silenced imaginary other. Structurally and semantically, the fantastic aims at dissolution of an order experienced as oppressive and insufficient (1981: 180).

Esa capacidad de oposición subversiva permite a las escritoras enfrentar la ideología dominante, lo que lleva en muchos casos a un porcentaje elevado de lo que podemos denominar como fantástico feminista, además de ir fraguando un canon de autoras de lo irreal que cada vez empieza a adquirir más relevancia. En todos estos aspectos se ahondará en este monográfico.

\section{LAS NARRADORAS ESPAÑOLAS ANTE LO FANTÁSTICO}

Lo afirmado con anterioridad ha contribuido a abrir nuevos caminos en el cultivo de lo fantástico y sus fronteras no miméticas, incluido el desarrollo de temas y formas narrativas que están ausentes o son poco exploradas en las obras de autores masculinos. Y, sea desde un cauce de fantástico femenino o de fantástico feminista, nos situamos ante obras siempre vinculadas a un marcado componente político e ideológico del que no huyen las estéticas de lo insólito. 
Uno de los debates todavía abierto en el ámbito de lo no mimético es el que trata de establecer una clasificación de sus categorías. Campra medita sobre dicha clasificación y la concibe como un mecanismo relevante en el proceso del conocimiento, aunque, a partir de un planteamiento crítico, constata las dificultades que conlleva cualquier intento de ordenación de los objetos culturales (2019: 79). A pesar de ello, la crítica especializada ha insistido en delimitar las tipologías de las estéticas de lo insólito, diferenciando con claridad entre lo fantástico, lo maravilloso, lo inusual y la ciencia ficción, sin olvidar otras categorías afianzadas en la tradición literaria, que serán explicadas y perfiladas en los artículos que se integran en este número especial dedicado a lo fantástico en creadoras españolas en el siglo XXI.

Todo ello se ha tenido en cuenta en el presente monográfico, en el que se confirma cómo lo fantástico no suele aparecer de manera aislada en la obra de las creadoras españolas, sino que se entrelaza en numerosas ocasiones con otras modalidades de lo irreal. En el caso de Cristina Fernández Cubas, comprobamos en el estudio de Patricia García cómo se vincula su cuentística tanto a lo fantástico como a lo gótico, el terror, lo grotesco, lo insólito y lo pseudo-fantástico. Por su parte, la singularidad de la narrativa de Pilar Pedraza obliga a Miguel Carrera Garrido a valorar sus vínculos con lo fantástico, el terror y el Female Gothic, entre otras posibles modalidades con las que se ha relacionado a la autora. Y Raquel Velázquez Velázquez demuestra la riqueza de modalidades de lo insólito que se conjugan en el universo minificcional de Julia Otxoa, desde lo maravilloso, a lo fantástico, lo simbólico-alegórico, lo distópico, lo surrealista, lo mitológico, lo absurdo, lo grotesco y lo esperpéntico. Atendiendo a las autoras de generaciones más jóvenes, mi aproximación a Patricia Esteban Erlés establece el hibridismo entre lo fantástico, el terror o el gótico sobrenatural, lo maravilloso y el realismo mágico. El colectivo Microlocas, a cuya obra se acerca Ada Cruz, ofrece ficciones breves fantásticas, pero también otras ubicadas en los márgenes de lo mimético y algunas propias de lo inusual. Y, aunque David Roas analiza exclusivamente el corpus fantástico de otras jóvenes escritoras, en algunas de sus publicaciones también podemos localizar hibridismo, con el cultivo del terror sobrenatural y de lo prospectivo.

Asimismo, un resorte muy significativo de la actual narrativa de autoría femenina española es el de la peculiar construcción y proyección semántica del espacio. La espacialidad literaria de la cuentística de Cristina Fernández Cubas es explorada en este monográfico por Patricia García, que introduce un enfoque todavía inédito en la crítica sobre esta autora. Desde la estética de lo liminal, parte de la teoría de los borderscapes para delimitar el dominio espacial en las tramas analizadas, aportando un prisma geocrítico a la figuración del espacio-santuario, en su doble dimensión de lugar de protección y de alienación. En mi artículo sobre Patricia Esteban Erlés pongo de relieve, a su vez, la impronta del espacio doméstico en sus narraciones y en las de autoras reconocidas dentro del canon hispánico femenino, sin olvidar el espacio del cuerpo, que no solo se trabaja en relación con Patricia Esteban Erlés, sino también con el colectivo Microlocas, con atención a sus microrrelatos fantásticos por parte de Ada Cruz. Ambos 
espacios, casa y cuerpo, reflejan el peso sobre la mujer de la imposición patriarcal y de las convenciones sociales derivadas de esta.

Además, en las ficciones de las escritoras españolas actuales de lo insólito destacan las voces narradoras de mujeres y el protagonismo femenino con una marcada agencia, que aporta novedosas miradas sobre la memoria, la familia, la infancia, la muerte, la otredad y el monstruo. Este último configura uno de los ejes más relevantes. En esa línea, sobresale el modo de abordar la monstruosidad de la realidad por parte de las creadoras. En algunas de ellas, como Pilar Pedraza y Patricia Esteban Erlés, a las que se añaden microrrelatos del colectivo Microlocas, asistimos al empleo de significativas actualizaciones, muy personales, del fantástico feminista que subvierte y transgrede rasgos del statu quo patriarcal a partir de la renovación del motivo del monstruo asociado a la mujer y al cuerpo de la mujer. En las poéticas de autoras más jóvenes interesa la propuesta de formas renovadoras en relación con la madre monstruo, la experiencia de la maternidad como conflicto y la niña monstruo, tal como sintetiza David Roas en su artículo, revelando cómo se ponen en cuestión las imposiciones a la mujer vinculadas a familia, maternidad y mundo infantil.

\section{CONCLUSIONES}

El presente monográfico pretende reivindicar la producción literaria realizada por las mujeres y, en particular, ensanchar nuestra comprensión de lo fantástico mediante el análisis de esta modalidad estética en las obras de autoría femenina. De ahí que los estudios se centren en obras de escritoras relevantes y consagradas, como Cristina Fernández Cubas (1945), Pilar Pedraza (1951) y Julia Otxoa (1953); de Patricia Esteban Erlés (1972), creadora con una trayectoria cada vez más consolidada; sin perder de vista el colectivo Microlocas —Eva Díaz Riobello (1980), Isabel González (1972), Teresa Serván (1974) e Isabel Wagemann (1972) - y la existencia de narradoras jóvenes que han entrado con fuerza en el panorama literario de lo fantástico español actual, entre ellas Ana Martínez Castillo (1978), Mónica Crespo (1980) y Aixa de la Cruz (1988).

La aproximación teórico-crítica a las citadas autoras permite demostrar la capacidad de lo fantástico como herramienta de conocimiento y de subversión, que se modela a través de diversas especificidades del corpus objeto de análisis. Con una llamativa inclinación por las formas breves - cuento y microrrelato mayoritariamente-, la distorsión de lo fantástico y sus fronteras no miméticas posicionan al lector ante una visión crítica y reflexiva sobre la condición humana, los avatares históricos y los seres marginados. La hipérbole, el humor o la ironía también resaltan en el contexto de testimonio y de denuncia de la falta de certezas y de las problemáticas sociales y de género en la sociedad actual, así como la renovación de los discursos tradicionales.

Los diversos artículos que engloban este estudio conjunto nos ofrecen conclusiones más amplias al respecto de esta concreta expresión fantástica femenina y de la literatura de umbrales que se localiza en las categorías estéticas de lo insólito. 


\section{REFERENCIAS BIBLIOGRÁFICAS}

Alemany BAy, C. y EudAVE, C., COORDS. (2020). [Monográfico] "Nuevas perspectivas sobre lo fantástico: escritoras hispanoamericanas y españolas (siglo XXI)". Brumal. Revista de Investigación sobre lo Fantástico VIII.1, 9-177. Disponible en línea: https://revistes.uab.cat/brumal/issue/view/v8-n1-2020 [10/12/2020].

Abrakova, M. G. (2004). El signo y el espejo. Una aproximación a lo fantástico femenino. México: Ediciones Eón.

Alpini, G. (2009). The Female Fantastic. Evolution, Theories and Poetics of Perversion. Fano: Aras Eidizioni.

Álvarez MÉNDEZ, N. (2013). “Introducción: Lo fantástico en la cultura española del siglo XXI”. Brumal. Revista de Investigación sobre lo Fantástico I.2, 195-200. Disponible en línea: https://revistes.uab.cat/brumal/article/view/v1-n2-alvarezmendez/pdf-es [10/12/2020].

CAMPRA, R. (2019). En los dobleces de la realidad. Exploraciones narrativas. León: Eolas.

Cranny-Francis, A. (1990). "Feminist Fantasy". En Feminist Fiction: Feminist Uses of Generic Fiction, 75-106. New York: St. Martin's Press.

GARcíA, P., ED. (2019). "Gender and the Hispanic Fantastic". Bulletin of Hispanic Studies 96.6, 569-682.

JACKSON, R. (1981). Fantasy, the Literature of Subversion. New York: New Accents.

LÓPEZ-PEllisA, T. y Ruiz GARZÓN, R. (2019). Insólitas. Narradoras de lo fantástico en Latinoamérica y España. Madrid: Páginas de Espuma.

MoErs, E. (1976). “Female Gothic”. En Literary Women: The Great Writers, 90-98. New York: Doubleday.

Richter, A. (1977). Le Fantastique féminin d'Ann Radcliffe à nos jours. Verviers: Marabout.

RoAS, D. Y MASSONI, A. (eds.) (2020). Las creadoras ante lo fantástico. Visiones desde la narrativa, el cine y el cómic. Madrid: Visor Libros.

RoAs, D.; Álvarez, N. Y GARCíA, P. (2017). "Narrativa 1980-2015”. En Historia de lo fantástico en la cultura española contemporánea (1900-2015), D. Roas (dir.), 195-214. Madrid / Frankfurt: Iberoamericana / Vervuert.

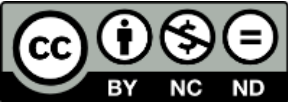

This work is licensed under a Creative Commons AttributionNonCommercial-NoDerivatives 4.0 International (CC BY-NC-ND).

Fecha de recepción: 12/01/2021

Fecha de aceptación: 16/03/2021

(C) UNED. Revista Signa 31 (2022), pp. 97-103

DOI: https://10.5944/signa.vol31.2022.32224

ISSN digital: 2254-9307. ISSN papel: 1133-3634 